\title{
Condición actual en la Reserva de la Biósfera Maya, tradición y reelaboración para una visión integrada de patrimonio mixto ${ }^{1}$
}

\section{Current Condition in the Maya Biosphere Reserve, Tradition and Reelaboration for an Integrated View of Mixed Heritage}

\author{
Oscar Antonio Quintana Samayoa \\ Facultad de Arquitectura, Universidad de San Carlos, Guatemala \\ Juan Antonio Siller Camacho \\ Seminario de Arquitectura Prehispánica, Facultad de Arquitectura, \\ Universidad Nacional Autónoma de México, México
}

\begin{abstract}
Resumen: Con el objetivo de estudiar sus condiciones, desde 1987 los autores han monitoreado los edificios prehispánicos ubicados en la Reserva de la Biósfera Maya (RBM) de Petén, Guatemala; también han dado seguimiento a áreas protegidas vecinas, como la Reserva de la Biósfera de Calakmul, en Campeche, México, y otras. Esta región de las tierras bajas centrales es conocida ahora por poseer un patrimonio doble: cultural y natural. Para la Reserva de la Biósfera Maya de Guatemala los datos indican que el manejo "ideal" mixto de patrimonios no ha resultado tan exitoso, pues los aspectos naturales y del medio ambiente prevalecen sobre los culturales. Por tal razón el presente trabajo intenta brindar una visión actualizada de la condición del patrimonio edificado en la RBM y revalorar el espíritu de un manejo de patrimonio mixto.
\end{abstract}

Palabras clave: Patrimonio mixto; Reserva de la Biósfera Maya; conservación; bienes inmuebles tangibles; arquitectura prehispánica.

\footnotetext{
${ }^{1}$ Una versión preliminar de este estudio fue expuesto en el XI Congreso Internacional de Mayistas, “Tradiciones y reelaboraciones”, celebrado en Chetumal, Quintana Roo, del 23 al 28 de junio de 2019.
} 
ABSTRACT: In order to study its conditions, since 1987 the authors have monitored the Prehispanic buildings located in the Maya Biosphere Reserve (MBR) in Petén, Guatemala; they have also analyzed neighboring protected areas such as the Calakmul Biosphere Reserve, in Campeche, Mexico, and others. This region of the Central lowlands is now known to possess a double heritage: cultural and natural. For the Maya Biosphere Reserve, in Guatemala, the data indicates that this mixed "ideal" management of heritage has not been successful, since the natural and environmental aspects prevail over the cultural ones. For this reason, the present work tries to give an updated view of the condition of the built heritage in the MBR and seeks to revalue the spirit of mixed management.

KEYwoRDs: Mixed heritage; Maya Biosphere Reserve; conservation; tangible immovable cultural heritage; Prehispanic architecture.

RECEPCIÓN: 18 de septiembre de 2019.

ACEPTACIÓN: 12 de diciembre de 2019.

Dor: https://doi.org/10.19130/iifl.ecm.2020.56.2.0006

\section{Introducción}

En 2019, el Consejo Nacional de Áreas Protegidas ${ }^{2}$ celebró 29 años de operaciones como responsable oficial de la Reserva de la Biósfera Maya. ${ }^{3}$ En el sitio arqueológico de Tikal se conmemoraron los 40 años de su declaratoria como el primer patrimonio mixto del mundo y los 60 años de la primera intervención restaurativa. Una particularidad de la RBM es su vecindad con otra zona importante de manejo, la Reserva de la Biósfera de Calakmul, en Campeche (México); juntas forman un gran conglomerado de importancia local, regional y mundial. A la vez, la Reserva de la Biósfera de Calakmul celebró 30 años de declaratoria (2019), 17 años de ser Patrimonio Cultural de la Humanidad y cinco como Patrimonio mixto. Durante el periodo Clásico maya, hace más de 1300 años, estas dos ciudades antagónicas protagonizaron una épica confrontación por el poder regional. En ese "juego de tronos", que duró más de 200 años, se involucraron muchas ciudades grandes, medianas y pequeñas, conflicto que determinó uno de los periodos culturales más florecientes del mundo maya. Una época llena de inscripciones con figuras de gobernantes y cautivos, embajadas reales, triunfos y derrotas que nos cuentan su historia.

El espacio territorial de tales eventos culturales se desarrolló en las llamadas "tierras bajas mayas del Centro". Definidas como una especie de bolsa, aislada de las costas, rodeada por montañas al este, sur y oeste (relieves entre 150 y 300 metros sobre el nivel del mar), al norte el paisaje se extiende y se une con las tierras bajas del Norte. El espacio se caracteriza por zonas de ríos estacionarios

\footnotetext{
2 En adelante CONAP.
}

${ }^{3}$ En adelante RBM. 
y permanentes, que drenan hacia el golfo de México o al mar Caribe; además hay extensas zonas inundables (humedales), suelo kárstico, sabanas, lagos y lagunas. Una diferencia notoria entre el norte y el centro es su flora: en Yucatán la selva es baja y espinosa con marcados cambios entre la época seca y la lluviosa, mientras que en la región central la selva es siempre verde, con variedad de árboles que llegan a más de $40 \mathrm{~m}$ de altura.

Podríamos decir que la historia cultural contemporánea inició en el año 1956 en Tikal, con las exploraciones del Museo de la Universidad de Pensilvania, a cargo de Edwin Shook, y en 1982 en Calakmul, por parte del Centro de Investigaciones Históricas y Sociales ${ }^{4}$ de la Universidad Autónoma de Campeche, ${ }^{5}$ con William J. Folan. Desde esas épocas a la actualidad, los actores y las circunstancias han cambiado. De las primeras excavaciones intensivas se han desarrollado múltiples proyectos de investigación sobre la naturaleza y los vestigios culturales de la zona. El territorio presenta una variedad de flora y fauna local mezclada con espacios urbanos prehispánicos que identifican esta parte del espacio maya, vestigios que le dan la categoría de Patrimonio mixto de la Humanidad.

\section{Antecedentes recientes de las reservas}

La condición aislada y el difícil acceso a esta parte del territorio maya, sin aparente riqueza para los conquistadores del siglo xvı y xviı, hizo que el área permaneciera por mucho tiempo sin mayores cambios: pocos habitantes, pocos caminos y una selva continua que escondía cientos de ciudades milenarias. A finales del siglo xIx y principios del xx, las futuras reservas de la biósfera fueron motivo de dos acciones: la extracción de madera (principalmente cedro y caoba) y el aprovechamiento del llamado "chicle de primera". Hubo en la selva varias concesiones de extracción de madera y algunos asentamientos que se dedicaban a recolectar el látex del árbol chicozapote (Manilkara zapota), producto que transportaban en mulas a centros de acopio. Fueron estos chicleros los primeros en recorrer sistemáticamente la selva: ellos buscaban bosques de chicozapote y encontraban ciudades abandonadas y en ruinas.

Los cambios en la continuidad paisajística comenzaron a acelerarse en las décadas de 1960 y 1970; en esa época, pobladores sin tierra, de otras regiones, llegaron al territorio. Entonces se pensaba que era una zona muy extensa y que se podía extraer sus recursos sin límite. En buena parte del siglo xx, el Estado guatemalteco sólo intervino en Tikal, dejando el resto de la futura RBM sin atención. Sin embargo, la falta de caminos mantuvo el estatus de abandono general del norte de Petén, condición que, a la vez, permitió el saqueo arqueológico. Por esos tiempos (los años cincuenta y sesenta) se reconoció mundialmente el valor

${ }^{4}$ En adelante CIHS.

5 En adelante UAC. 
artístico maya, categoría que indujo a un expolio de bienes muebles de arte maya que continúa hasta ahora.

En la parte mexicana, entre las décadas del cuarenta al sesenta sólo había, en la región sur de Campeche, algunos caminos de terracería usados por los madereros. Durante los sesenta se completó el camino de Xpujil a Chetumal, mejorando una conexión oeste-este paralela a la frontera con Petén (carretera EscárcegaChetumal). Luego llegaron más colonos y la ganadería. En el año 1989 se formuló la Reserva de la Biósfera de Calakmul, y en 1993 Calakmul se incluyó dentro del programa "El Hombre y la Biosfera" de la unEsCO ${ }^{6}$ (ampliado en el año 2006). En 1996 se perfiló el municipio de Calakmul (1,383,911 habitantes), territorio que incluye ahora la Reserva de la Biósfera.

En la parte de Guatemala, las actividades puntuales, iniciadas en 1955 en Tikal, continuaron sin pausa. En 1987, además de trabajarse allí, se amplió la investigación a otros sitios arqueológicos vecinos. En esa época se realizaron varios viajes de reconocimiento por la selva petenera. La intención de los recorridos fue crear parámetros de condiciones del patrimonio edificado prehispánico. Afortunadamente, el primer recorrido, realizado en 1987, fue publicado en los Cuadernos de Arquitectura Mesoamericana (Siller y Quintana, 1989). Desde esa época hemos usado como parámetro los indicadores publicados por el Seminario de Arquitectura Prehispánica ${ }^{7}$ de la Facultad de Arquitectura ${ }^{8}$ de la Universidad Nacional Autónoma de México, ${ }^{9}$ datos que nos han permitido evaluar y monitorear el comportamiento del patrimonio edificado en la RBM, actividad que continúa hasta la fecha.

Desde el año 2012, hemos expuesto en diversos foros internacionales nuestra visión de la tradición y la reelaboración de esta parte del territorio maya: el Congreso del Comité Internacional del Patrimonio Arqueológico (Cuzco, 2012), la Primera Mesa Redonda del Mayab (Mérida, 2014), el Primer Congreso Internacional de Patrimonio Mixto Natural y Cultural, "La Carta de Puebla" (Puebla, 2016), el Primer Coloquio Los Mayas en el $\mathrm{INAH}^{10}$ (México, 2018) y el XI Congreso Internacional de Mayistas (Chetumal, 2019).

En cuanto a la condición actual de las Reservas de la Biósfera (Calakmul y Maya), podemos constatar que, a partir de la década de 1990, el aspecto natural y ambiental ha adquirido gran relevancia y protagonismo. Las instituciones oficiales como la Comisión Nacional de Áreas Naturales Protegidas ${ }^{11}$ en México y la ConAP en Guatemala, por ley, son las responsables de las áreas protegidas. Pero en muchos casos dichas reservas territoriales cuentan también con un patrimonio cultural. La gestión cultural ha quedado relegada a segundo plano en las decisiones de los planes de manejo (liderados por especialistas en patrimonio natural).

\footnotetext{
${ }^{6}$ Organización de las Naciones Unidas para la Educación, la Ciencia y la Cultura.

${ }^{7}$ En adelante SAP.

${ }^{8}$ En adelante FA

${ }^{9}$ En adelante UnAm.

${ }^{10}$ Instituto Nacional de Antropología e Historia.

11 En adelante CONANP, establecida en el año 2000.
} 
Por esa razón en este trabajo presentamos una visión de la RBM desde el punto de vista del patrimonio inmueble tangible (sitios y edificios prehispánicos).

Debemos aclarar que el patrimonio construido prehispánico (patrimonio inmueble o tangible) de la RBM es uno de los varios patrimonios culturales que posee la gran reserva. Por ejemplo, hay bienes culturales transportables (patrimonio mueble tangible) de carácter cotidiano o ceremonial, como cerámica, artefactos de piedra verde, objetos de hueso y concha o estelas y altares tallados en piedra. Una particularidad especial, en esta zona del territorio maya, es la memoria epigráfica. Una extensa evidencia de textos escritos preserva relatos, registrados en piedra, estuco, pintura y otras superficies, sobre acontecimientos históricos de los gobernantes sucedidos durante el periodo Clásico.

Por otro lado, la RBM también posee cultura viva con poblados históricos y arquitectura vernácula, como en Uaxactún, San José y Carmelita. Y posee asimismo patrimonio intangible: leyendas de los antepasados, tradiciones de los chicleros y sus campamentos en la selva o historias de notorios saqueadores de tesoros mayas, aunado a una tradición culinaria y al desarrollo de las comunidades mayas itzáes.

\section{Administración actual del norte de Petén}

Por su condición "aislada” y de difícil acceso, en la primera parte del siglo xx, el norte del departamento permaneció desatendido. En 1948 se instaló, en Petén, un destacamento militar, y una década después, en 1959, se formó la Empresa de Fomento y Desarrollo de Petén. ${ }^{12}$ Esta organización decidió dejar la parte norte del departamento como reserva forestal ("Reserva Forestal FYDEP”, arriba del paralelo 17 grados 10 minutos); tal acción impidió que se otorgaran tierras a colonos en dicho territorio. En 1985 desapareció el FYDEP. Cuatro años después el norte de Petén pasó a formar parte del Sistema Nacional de Áreas Protegidas de Guatemala ${ }^{13}$ y en 1990 se decretó la Reserva de la Biósfera Maya, confirmando la parte norte de Petén para un uso forestal y reserva natural. Dentro de este nuevo esquema quedó Tikal como el primer Parque Nacional de Centroamérica (1955). Por su antiguiedad, es la única zona protegida administrada exclusivamente por el Ministerio de Cultura y Deportes ${ }^{14}$ a través del Instituto de Antropología e Historia ${ }^{15}$ de Guatemala.

Tikal fue el primer sitio, en todo Petén, en tener un plan maestro, instrumento que entró en operación en 1972. Este documento de planificación incluyó, además del cuadrado del parque (24 por $24 \mathrm{~km}$ ), una serie de infraestructura

\footnotetext{
12 En adelante FYDEP.

${ }^{13}$ En adelante SIGAP. Ley de áreas protegidas, decreto No. 4-89 del Congreso de la República de Guatemala.

14 En adelante MCD.

15 En adelante IDAEH.
} 
necesaria para visitar el sitio: carretera asfaltada Flores-Tikal, aeropuerto internacional en Santa Elena, instalaciones de servicio en el parque y otros (sobre el origen, desarrollo e importancia de Tikal y sus vecinos puede verse Quintana 2013a; 2013b; 2014).

De una sola área protegida declarada "Parque Nacional Tikal" (territorio de 57,583 ha), se pasó a tres parques nacionales: Tikal, Mirador-Río Azul y Yaxhá, Nakum y Naranjo, con 195,943 ha), dos biotopos (zonas núcleo, con 89,450 ha protegidas), dos corredores biológicos, áreas de concesiones forestales industriales y comunitarias, ${ }^{16}$ más una zona de amortiguamiento. ${ }^{17}$ La RBM cuenta ahora con 2,083,495 ha, co-administradas entre MCD-IDAEH, USAC, ${ }^{18}$ CONAP y cuatro jurisdicciones municipales (1990) ${ }^{19}$ (Figura 1).

En la vecina Reserva de la Biósfera de Calakmul, las bases y conceptos de una reserva fueron desarrollados por los investigadores del CIHS de la UAC (1974-1982). Luego, en 1989, fue declarada Reserva de la Biósfera y en el año 2002 como patrimonio cultural de unEsCo, y como Patrimonio Mixto en 2014. En el expediente de UNESCO, Calakmul tiene registrados 38 centros arquitectónicos mayas en cuatro diferentes rangos: doce centros mayores, nueve centros medios, seis centros menores y once sitios pequeños. Además, incluye un mosaico de comunidades de bosque tropical con redes ecológicas y tróficas complejas (86 especies de mamíferos, 282 de aves, 50 de reptiles, 400 de mariposas y 73 tipos de orquídeas salvajes).

En el mundo hay 39 sitios mixtos; en América tan sólo hay seis, más una isla administrada por Estados Unidos. El primero en el mundo fue Tikal, en Guatemala (26 de octubre 1979); en Perú, Machu Picchu (1983) y el Parque Nacional de Río Abiseo (1990-1992); en Hawái, Estados Unidos, Papahänaumokuäkea (2010); en Jamaica, las Montañas Azules y de John Crow (2015), y en Campeche, México, la antigua ciudad maya y los bosques tropicales protegidos de Calakmul (20022014) y el Valle de Tehuacán-Cuicatlán, hábitat originario de Mesoamérica, en 2018 (para las definiciones y términos sobre patrimonio cultural, natural y mixto, véase UNESCO, 2012).

Guatemala todavía no cuenta con un claro y detallado inventario cultural para la RBM; tampoco una visión estratégica. En la actualización del plan maestro de la RBM (2016) se habla de tres categorías (sitios centrales, periféricos y menores), pero el plan sólo caracteriza el primer nivel (28 sitios centrales).

Los elementos y valores de ambas reservas son similares, condición que hace posible, por ser vecinas, formar planificaciones conjuntas bajo una visión mayor llamada "Selva Maya", un espacio más amplio que incluye 150 áreas protegidas de diferentes categorías de manejo en México, Belice y Guatemala.

16 zum: Zona de Uso Múltiple.

17 ZAM: Zona de Amortiguamiento.

${ }^{18}$ Universidad de San Carlos, Guatemala.

${ }^{19}$ Con referencia a la RBM, véase Reserva de la Biósfera Maya, Plan Maestro, segunda actualización, diciembre 2015, documentos técnicos núms. 20, 21, 22 y 23-2016. 

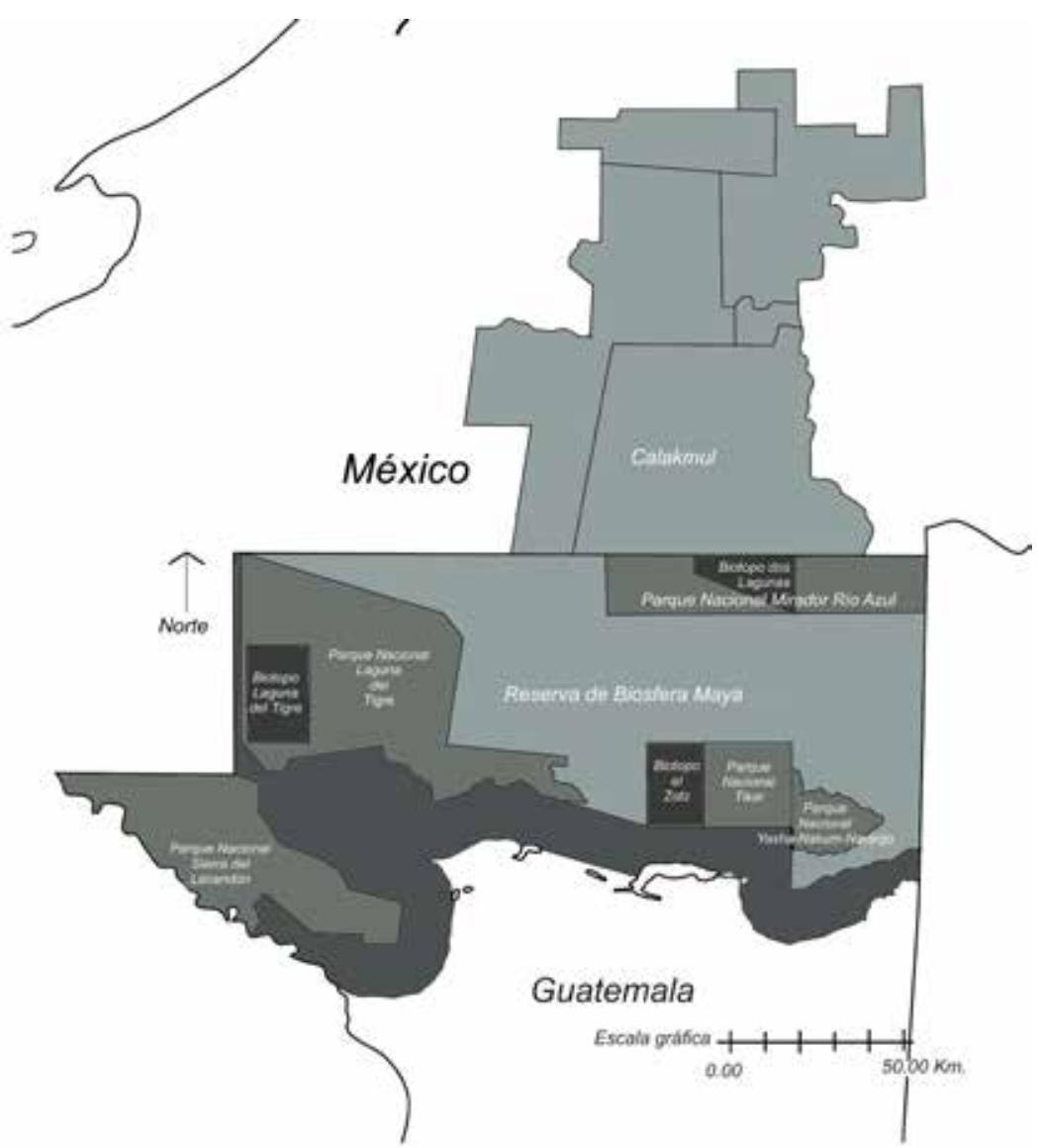

Figura 1. Mapa de ubicación de la Reserva de la Biósfera Maya (RBM)

y de la Reserva de la Biósfera de Calakmul, indicando la condición administrativa de la RBM (modificado de conAP, 2016: Mapas 1 y 2, dibujo de Raúl Noriega).

En la parte guatemalteca, luego de haberse aprobado el SIGAP (1989) y la RBM (1990), siguió la preparación de los primeros planes maestros. El primer plan de la Rвм fue en 1992-1997; luego se actualizó durante 2001-2006 y en diciembre de 2015 se aprobó la segunda actualización. ${ }^{20}$ Adicionalmente hay otros planes complementarios como el Plan Maestro del Parque Nacional Yaxhá, Nakum, Naranjo (1999-2003, 2006-2010, y la última actualización CATIE ${ }^{21}$ 2013-14, aprobado en el año 2019); el Plan Maestro de Tikal (2004-2008); el Plan Maestro del Parque Nacional Mirador-Río Azul y Biotopo Protegido Naachtún Dos Lagunas (20092013); el Plan Maestro del Parque Nacional Sierra del Lacandón (2005-2009, espa-

\footnotetext{
20 ConAP, documentos técnicos núms. 20, 21, 22 y 23-2016.

${ }^{21}$ Centro Agronómico Tropical de Investigación y Enseñanza.
} 
cio co-administrado desde el año 1999 por la Fundación Defensores de la Naturaleza); el Plan Maestro del Parque Nacional Laguna del Tigre y Biotopo Laguna del Tigre (2007-2011), y otros. Todos los planes fueron formulados por onGs ${ }^{22}$ locales o internacionales que se dedican al patrimonio natural (TNC, ${ }^{23}$ WCS, ${ }^{24}$ CATIE, UICN, ${ }^{25}$ ONCA ${ }^{26}$ y otros). La metodología utilizada para los planes fue diseñada para áreas protegidas naturales y adaptada para la RBM (USAID, ${ }^{27}$ método para la conservación de áreas $\left.\mathrm{PCA},{ }^{28} \mathrm{GIZ},{ }^{29} \mathrm{EAPC}^{30}\right)$.

El ConAP, para evaluar los aciertos, retos y desafíos de 29 años de administración y manejo de la RBM, ordena en cuatro tendencias sus informes oficiales: 1) voluntad y compromiso del Estado; 2) estabilidad en el sector este de la RBM; 3) los retos continúan, y 4) financiamiento del conAP. El último informe del conAP fue preparado en 2017, "Monitoreo de la gobernabilidad en la RBM" (CONAP-wCS, 2018). El documento señala que la parte este de la RBM es la más estable (1.1 millones de ha); es un espacio co-administrado por nueve concesionarios forestales comunitarios, dos concesionarios forestales industriales, tres parques nacionales y un corredor biológico. La parte oeste de la RBM (según el informe de ConAP-wCS, 2018: 32), es la más conflictiva: el Parque Nacional Laguna del Tigre (337,899 ha) y el biotopo protegido Laguna del Tigre. En mejores condiciones, según el referido informe de monitoreo, está la cuenca del río Usumacinta (Parque Nacional Sierra del Lacandón).

El IDAEH, que fue la primera institución estatal en tener presencia física en la futura RBM, ha sido administrativamente, con excepción de Tikal, relevada por el CONAP. Aunque el IDAEH es parte del CONAP, su participación en las decisiones del Consejo ha sido muy reducida. Es claro que el tema del ambiente y los recursos naturales son los protagonistas actuales de la RBM. Las decisiones del ConAP son tomadas por siete miembros (cuatro del sector naturaleza y ambiente, del IDAEH, las municipalidades y el Instituto de Turismo de Guatemala). ${ }^{31}$

Así, el tema cultural queda relegado a: 1) Tikal, importante enclave de interés político en el departamento; 2) el interés de universidades extranjeras en excavaciones arqueológicas dentro de la RBM, y 3) pocos sitios habilitados con alguna infraestructura para recibir visitantes (Tikal, Uaxactún y Yaxhá-Topoxté).

Según el último informe de monitoreo y gobernabilidad (ConAP-WCS, 2018: 32), en el año 2017 Tikal fue visitado por más de 300 mil personas; Yaxhá, en segundo lugar, contó con 27,000 visitantes. Entre otros sitios arqueológicos monitoreados

22 Organizaciones No Gubernamentales.

23 The Nature Conservancy.

${ }^{24}$ Wildlife Conservation Society.

25 Unión Internacional para la Conservación de la Naturaleza.

26 Organismo Nacional de Certificación Agroalimentario, A.C.

${ }^{27}$ United States Agency for International Development.

28 Planificación para la Conservación de Áreas.

${ }^{29}$ Deutsche Gesellschaft für Internationale Zusammenarbeit (Sociedad Alemana para la Cooperación Internacional).

30 Estándares abiertos para la práctica de la conservación.

31 En adelante InGUAT. 
están Tayasal, El Mirador (fuertemente promovido por el proyecto de investigación Cuenca Mirador), Tintal, La Florida, Nakbé, Ixlú, El Perú y otros. En el mismo año, el sitio arqueológico de Calakmul, en Campeche, registró 39,000 visitantes.

El MCD, a pesar de poseer en la RBM la mayor reserva arqueológica protegida de Guatemala, no ha podido tener una visión de su patrimonio. El MCD necesita crear una agenda estratégica que reactive la gestión cultural y se integre, de verdad, con el sector naturaleza procurando un manejo de patrimonio mixto, con categoría de Patrimonio de la Humanidad.

\section{Antecedentes culturales en la RBM}

El conocimiento de las ciudades mayas en la futura RBM también inició relativamente tarde. A finales del siglo xix e inicios del xx, en el norte de Petén se conocían cinco sitios arqueológicos (Tayasal, Motul de San José, San Clemente, Topoxté y Tikal). Luego, a partir del año 1904, aumentó el registro de sitios (Yaxhá, Naranjo, Nakum y otros). El primer documento que integró los avances del conocimiento arqueológico en Petén fue elaborado por Sylvanus Griswold Morley (1937-1938). Morley estuvo también en Calakmul (1932), sitio descubierto en el año 1931 por Cyrus L. Lundell. Como mencionamos antes, Calakmul pasó sin mayor interés por varias décadas, hasta que los investigadores del CIHS de la UAC iniciaron, a partir del año 1974, reconocimientos sistemáticos en la selva campechana. El Seminario de Arquitectura Prehispánica de la FA, UnAM, también realizó prospecciones en esta zona, en 1985 en la región Río Bec, en 1986 en la región Chenes y en 1987 en Petén (Siller, 1991a; 1991b; para mayor información sobre los sitios arqueológicos en la RBM véase Quintana, 2015 y Quintana y Siller, 2019).

\section{Intervenciones en edificios prehispánicos de la RBM}

La primera acción sobre edificios de la futura RBM sucedió en Uaxactún (19271937); en este caso, la institución Carnegie de Washington desmanteló edificios prehispánicos dejándolos luego desarticulados, abiertos y abandonados. Los argumentos para tal destrucción fueron: que Uaxactún estaba aislado en la selva, que no había caminos y que allí nunca llegaría el turismo... Luego fue Tikal ("Proyecto Tikal" del Museo de la Universidad de Pensilvania, 1956-1969). Los trabajos preparatorios para el "Proyecto Tikal" iniciaron con la apertura en la selva de una pista de aterrizaje (1951), y luego con la delimitación de un cuadrado protegido (Parque Nacional Tikal, 1955). En esa época, aunque, el territorio seguía estando aislado en la selva, sin comunicación vial, se pensó en un proyecto de investigación y preparación de partes de edificios para un uso turístico. Actividad que ha proseguido hasta ahora con diferentes acciones, consecuencias y resultados (Quintana, 2014). 
Como mencionamos antes, en el año 1987 inició, partiendo de Tikal, una serie de recorridos por la futura reserva. El equipo de trabajo para los reconocimientos de campo contó con el apoyo científico de la FA y el SAP de la UNAM. Paul Gendrop (director del seminario) contribuyó con esta iniciativa enviando a sus alumnos a Tikal, apuntalando así el plan de apertura del parque. Además, en forma oficial el MCD solicitó apoyo internacional para descentralizar Tikal. En respuesta, el gobierno alemán envió a un consultor del Instituto Arqueológico Alemán (director de la Comisión de Arqueología General y Comparada-KAVA-DA ${ }^{32}$ Wolfgang Wilhelm Wurster). Wurster ayudó al IDAEH a formular un fondo de Cooperación Bilateral (Alemania-Guatemala), que comenzó a operar en 1994. Con estos fondos se trabajó, desde el año 1994 al año 2006, el llamado "triángulo cultural Yaxhá, Nakum, Naranjo" (Quintana, 2013b).

En esa época se formuló el "Plan de Conservación y Manejo de Monumentos Culturales de Petén" (Quintana y Siller, 1992). Las acciones del plan se dividieron en dos: una, fue ampliar el radio de atención a edificios en peligro de colapso alrededor de Tikal (fuera de su cuadrado histórico), y la otra fue a nivel regional y tuvo el propósito de elaborar un registro del potencial cultural del territorio alrededor de Tikal ("Programa de Rescate"). Este programa realizó trabajos de documentación, rescate y planificación regional con acciones de reconocimiento de ciudades mayas por espacio de 18 años (1987-2005, Quintana, 2013b; 2015). El resultado de los recorridos por la selva fue publicado por el Instituto Arqueológico Alemán (Quintana y Wurster, 2001 y Quintana, 2013a). Datos actualizados fueron publicados recientemente (Quintana y Siller, 2019).

Por otro lado, la iniciativa privada también ha aportado acciones en la RBM. La Fundación Patrimonio Cultural y Natural Maya ${ }^{33}$ invierte fondos, desde el año 2005, en la investigación arqueológica en la RBM. A partir del año 2016 se conformó un consorcio con varios investigadores para unificar una metodología usando como base imágenes obtenidas a través de la tecnología cartográfica aérea LiDAR. Con ello pacunam formula, para el IDAEH, un nuevo Plan de Conservación y Puesta en Valor Sostenible de la RBм. Con relación al patrimonio natural, desde el año 2006 actúa en la zona el Programa Selva Maya-UICN/KfW ${ }^{34}$ con acciones que involucran sectores de la RBM, partes de Campeche y Quintana Roo, en México, y Belice.

\section{Primera síntesis, edificios mayas en la RBM}

El primer informe, sobre 14 ciudades en la RBм, fue publicado en 1989 (Siller y Quintana, 1989). En este documento se calificó el estado de conservación y prioridad con base en dos indicadores: 1) arquitectura visible en peligro (con tres

\footnotetext{
32 Kommission für Allgemeine und Vergleichende Archäologie des Deutschen Archäologischen Institutes in Bonn.

33 En adelante PACUNAM.

${ }^{34}$ Banco alemán con sede en Frankfurt: Kreditanstalt für Wiederaufbau.
} 
variables) y 2) cantidad de saqueos arqueológicos (también con tres variables). $\mathrm{Al}$ combinar estos dos indicadores y compararlos con los datos actuales tenemos una visión de lo sucedido en la RBM.

\section{Sitios centrales, periféricos y menores}

En el año 2013 se actualizó el Plan Maestro de la RBM (ConAP, documento técnico núm. 20-2016: 138-139), actual instrumento rector en el ámbito de su planificación. El plan clasificó los yacimientos mayas en tres categorías: sitios centrales, sitios periféricos y sitios menores. Este documento técnico sólo caracterizó a los llamados "sitios centrales", o sea, los yacimientos grandes o estratégicos. Para las otras categorías no se da mayor información. El documento hace referencia a varios sitios periféricos, pero no se da una cuantificación sobre este rango. Igual sucede con los sitios menores; no hay datos cuantitativos sobre tal condición del patrimonio edificado.

En general, el plan hace referencia a 425 sitios arqueológicos en la RBM y se considera que posiblemente existen más de 1,000 (CONAP, 20-2016: 139). El criterio usado para definir los 28 sitios centrales es el resultado de varios talleres de consulta, por lo tanto, aparecen en la lista yacimientos emblemáticos (Tikal o Yaxhá); otros fueron escogidos por su importancia histórica y epigráfica (La Corona o El Perú), unos por poseer investigación arqueológica avanzada (Holmul o El Mirador) y algunos por contar con arquitectura expuesta en peligro de colapso (La Honradez o Kinal), etcétera.

\begin{tabular}{|l|l|}
\hline Sitios centrales & Plan Maestro RBM 2016 \\
\hline 1. Río Azul-Kinal & 15. Motul de San José \\
\hline 2. San Bartolo-Xultún & 16. Naachtún \\
\hline 3. Holmul-Cival & 17. El Mirador-Nakbé \\
\hline 4. La Honradez & 18. El Tintal-El Porvenir \\
\hline 5. Naranjo & 19. Wakná \\
\hline 6. Tzikintzakán & 20. Las Guacamayas \\
\hline 7. Yaxhá-Topoxté & 21. La Corona \\
\hline 8. Nakum & 22. El Perú \\
\hline 9. San Clemente & 23. Laguna Perdida \\
\hline 10. La Blanca & 24. La Joyanca \\
\hline 11. Sacpetén & 25. El Reinado \\
\hline 12. Tikal & 26. La Florida \\
\hline 13. Uaxactún & 27. La Pasadita-Tecolote \\
\hline 14. El Zotz & 28. Piedras Negras \\
\hline
\end{tabular}

Cuadro 1. Sitios centrales de la RBM, según el Plan Maestro

(CONAP, 2016, t. 1: Mapa 15). 


\section{Monitoreo de sitios en la RBM}

En este trabajo se toman en cuenta los "indicadores cualitativos" del Plan 2016 incluyendo otros que puedan ser medibles (arquitectura visible y cantidad de saqueos). Valores que, si se tienen antecedentes publicados, pueden constituir una base de datos para comprobar si ha existido un manejo eficiente o no en la RBM. En este sentido, los yacimientos "agregados" pueden ser considerados, siguiendo la idea del plan actual, como "sitios periféricos". Con este criterio se seleccionaron 60 yacimientos (incluyendo los 28 sitios centrales considerados en el Plan de 2016); o sea, tenemos 28 sitios centrales (del plan) más 32 sitios periféricos (Figura 2, Cuadro 2).

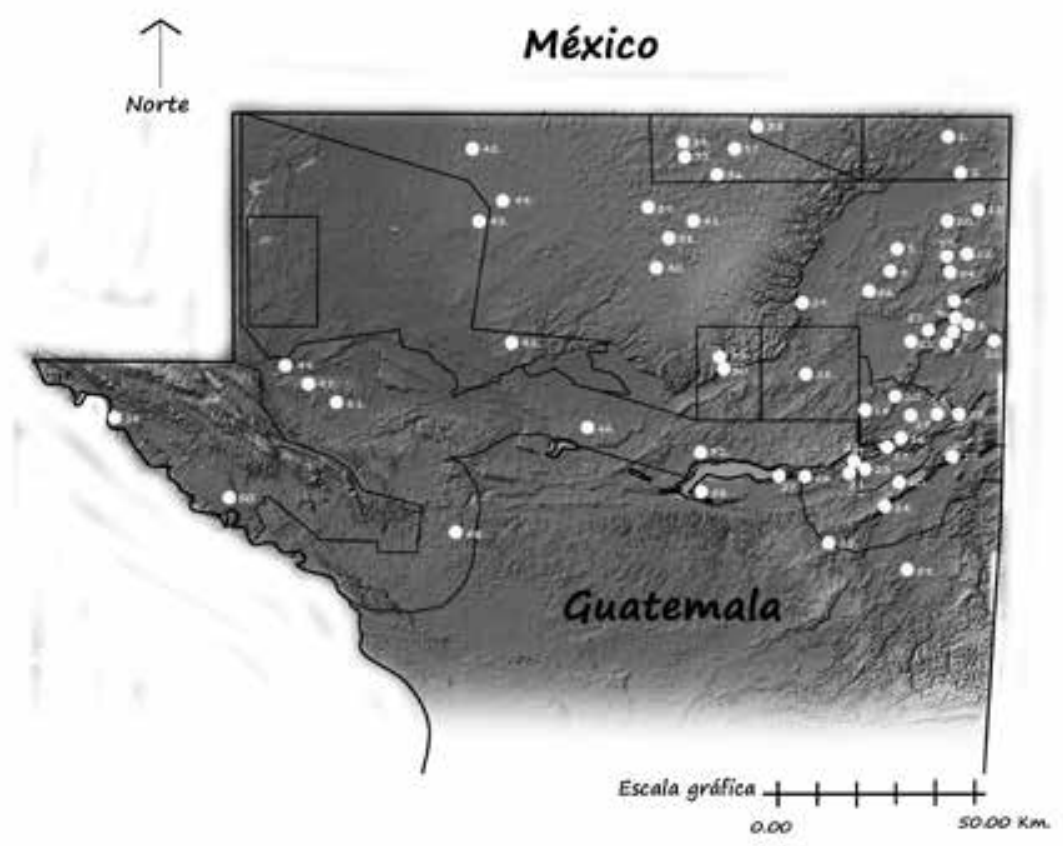

Figura 2. Sitios diagnósticos. El mapa incluye como número 61 al sitio San Diego-La Gloria, considerado por el PMRBM-2016, que no presenta arquitectura expuesta pero sí un importante petrograbado (datos de Oscar Quintana, dibujo de Raúl Noriega).

El Cuadro 2 muestra 60 yacimientos seleccionados como posibles indicadores de la condición del patrimonio edificado en la RBM. Las primeras cuatro casillas identifican la muestra disponible con un número correlativo de 1 a 60 . La siguiente, el número correspondiente al PMRBM-2016. ${ }^{35}$ Sigue el nombre del sitio

35 Plan Maestro de la Reserva de la Biósfera Maya. 


\begin{tabular}{|c|c|c|c|c|c|c|c|c|c|}
\hline & & CIUDADES MA & AYAS, R & $\mathrm{M} 20$ & & & & & \\
\hline No. & & NOBRE & TIPO & PARTE & STE & cuenca & parte & sierra & Mopán \\
\hline & PMRBM & sitio & uso & Este & centro & Los Lagos & Oeste & Lacand. & \\
\hline 1 & 1 & RIO AZUL & $\mathrm{PN}$ & $x$ & & & & & \\
\hline 2 & 1 & KINAL & $\mathrm{PN}$ & $x$ & & & & & \\
\hline 3 & 2 & SAN BARTOLO & zUM & $\mathrm{x}$ & & & & & \\
\hline 4 & 2 & XULTÚN & ZUM & $x$ & & & & & \\
\hline 5 & 3 & HOLMUL & zUM & $x$ & & & & & \\
\hline 6 & 3 & CIVAL & zUM & $\mathrm{x}$ & & & & & \\
\hline 7 & & SUFPICAYA & ZUM & $\mathrm{x}$ & & & & & \\
\hline 8 & & PERÚ-HOLMUL & ZUM & $\mathrm{x}$ & & & & & \\
\hline 9 & & WITZNÁ & zUM & $x$ & & & & & \\
\hline 10 & 4 & LA HONRADEZ & ZUM & $\mathrm{x}$ & & & & & \\
\hline 11 & & CHOCHKITAM & zUM & $\mathrm{x}$ & & & & & \\
\hline 12 & & XMAKABATÚN & zUM & $x$ & & & & & \\
\hline 13 & 5 & NARANJO SA'AL & $\mathrm{PN}$ & $\mathrm{x}$ & & & & & \\
\hline 14 & & EL PITAL & $\mathrm{PN}$ & $\mathrm{x}$ & & & & & \\
\hline 15 & 6 & TZIKINTZAKÁN & ZUM & & & & & & $x$ \\
\hline 16 & 7 & YAXHÁ & $\mathrm{PN}$ & & & $x$ & & & \\
\hline 17 & 7 & TOPOXTÉ & $\mathrm{PN}$ & & & $x$ & & & \\
\hline 18 & & NARANUITO & $\mathrm{PN}$ & $\mathrm{x}$ & & & & & \\
\hline 19 & & POZA MAYA & $\mathrm{PN}$ & $\mathrm{x}$ & & & & & \\
\hline 20 & 8 & NAKUM & $\mathrm{PN}$ & $\mathrm{x}$ & & & & & \\
\hline 21 & 9 & SAN CLEMENTE & ZAM & & & $x$ & & & \\
\hline 22 & & ZAPOTE COROZAL & ZAM & & & $x$ & & & \\
\hline 23 & & COROZAL TORRE & ZAM & & & $x$ & & & \\
\hline 24 & 10 & LA BLANCA & ZAM & & & & & & $\mathrm{X}$ \\
\hline 25 & & CHILONCHE & FUERA & & & & & & $\mathrm{X}$ \\
\hline 26 & 11 & SACPETEN & ZAM & & & $x$ & & & \\
\hline 27 & & ixú & ZAM & & & $x$ & & & \\
\hline 28 & 12 & TIKAL & $\mathrm{PN}$ & $\mathrm{x}$ & & & & & \\
\hline 29 & 13 & UAXACTÚN & zUM & $x$ & & & & & \\
\hline 30 & 14 & EL ZOTZ & BIOT & $\mathrm{x}$ & & & & & \\
\hline 31 & & EL DIABLO & BIOT & $\mathrm{x}$ & & & & & \\
\hline 32 & 15 & MOTUL & ZAM & & & $x$ & & & \\
\hline 33 & 16 & NAACHTÚN & BIOT & $\mathrm{x}$ & & & & & \\
\hline 34 & 17 & EL MIRADOR & $\mathrm{PN}$ & & $x$ & & & & \\
\hline 35 & & LA MUERTA & $\mathrm{PN}$ & & $x$ & & & & \\
\hline 36 & 17 & NAKBE & $\mathrm{PN}$ & & $x$ & & & & \\
\hline 37 & & LA MURALLA & $\mathrm{PN}$ & & $x$ & & & & \\
\hline 38 & 18 & EL TINTAL & zUM & & $x$ & & & & \\
\hline 39 & 18 & EL. PORVENIR & zUM & & $x$ & & & & \\
\hline 40 & & LA FLORIDA & zUM & & $x$ & & & & \\
\hline 41 & 19 & WAKNNÁ & ZUM & & & & & & \\
\hline 42 & 20 & LAS GUACAMAYAS & ZUM & & & & & & \\
\hline 43 & 21 & LA CORONA & zUM & & & & & & \\
\hline
\end{tabular}

Cuadro 2. Ciudades mayas en la RBM, condición del patrimonio edificado. 


\begin{tabular}{|c|c|c|c|c|c|c|c|c|c|}
\hline 44 & & EL ACHIOTAL & ZUM & & & & & & \\
\hline 45 & 22 & PERÚ WAKÁ & PN & & & & & & \\
\hline 46 & 23 & LAGUNA PERDIDA & ZAM & & & & & & \\
\hline 47 & 24 & LA JOYANCA & ZAM & & & & & & \\
\hline 48 & 25 & EL REINADO & ZAM & & & & & & \\
\hline 49 & 26 & LA FLORIDA & ZAM & & & & & & \\
\hline 50 & 27 & TECOLOTE & PN & & & & & & \\
\hline 51 & 28 & PIEDRAS NEGRAS & PN & & & & & & \\
\hline 52 & & EL PILAR & TRÁMITE & & & & & & \\
\hline 53 & & EL SUSTO MIRADO & ZUM & & & & & & \\
\hline 54 & & EL SUSTO & ZUM & & & & & & \\
\hline 55 & & EL DANTO & ZUM & & & & & & \\
\hline 56 & & SAN MÁXIMO & ZUM & & & & & & \\
\hline 57 & & LAS VENTANAS & ZUM & & & & & & \\
\hline 58 & & TAYASAL & FUERA & & & & & & \\
\hline 59 & & UCANAL & FUERA & & & & & & \\
\hline 60 & & HOLTÚN & ZAM & & & & & & \\
\hline & & & & 28 & 8 & 11 & 7 & 2 & 4 \\
\hline
\end{tabular}

Cuadro 2. Ciudades mayas en la RBM, condición del patrimonio edificado (continuación).

arqueológico y luego la condición administrativa actual o espacio en que se encuentra (PN, corresponde a parque nacional, zUM: zona de usos múltiples, zAM: zona de amortiguamiento; вІот: biotopo, y un calificativo para los sitios que no están dentro del área de la RBM, como: fuera).

La segunda parte de la tabla indica la posición del yacimiento dentro de la RBM: la parte este está dividida en dos: este y central. Cada segmento tiene a su vez diferentes sub-divisiones, por ejemplo, la parte este está formada por la cuenca río Ixcán, cuenca río Holmul y Área Tikal. El segmento central corresponde a la Cuenca Mirador. La parte oeste atañe al sistema de la Laguna del Tigre. Una franja sur, en donde se encuentra la zona de amortiguamiento, redes viales, poblados y el avance de la frontera agrícola está conformada por tres espacios: al suroeste, la Cuenca del río San Pedro Mártir; al centro, la Cuenca de los Lagos, y al sureste, la Cuenca Mopán. Por otro lado, al oeste, siguiendo la Cuenca del río Usumacinta (del lado guatemalteco), está la porción Sierra Lacandón (Figura 3).

Para cada yacimiento hay una "ficha técnica" con datos de su condición actualizada. En estos registros se anotan, siguiendo los indicadores establecidos en 1987 (arquitectura en peligro y cantidad de saqueos), el estado de condición de cada uno, información que luego puede ser cotejada con otras publicaciones o nuevos trabajos de campo.

Treinta y seis ciudades están dentro de la llamada parte este (60\% de la muestra), considerada por ConAP como la zona más estable de la RBM. En ella está Tikal y por consecuencia las acciones del Programa de Rescate (1987-2005). 


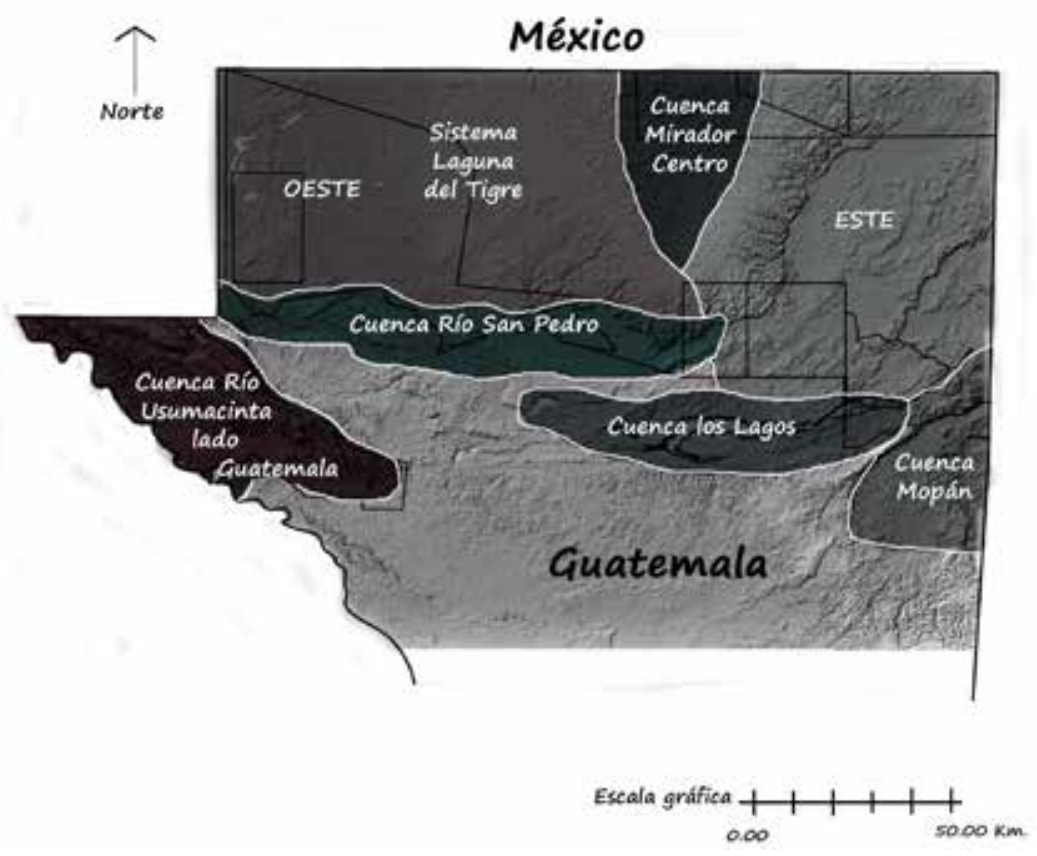

Figura 3. Distribución espacial de ciudades mayas en el norte de Petén (datos de Oscar Quintana, dibujo de Raúl Noriega).

La parte Sureste de la RBM, coincide con las redes viales actuales y nuevos poblados, en espacios de las zam y zum; tiene una muestra de 11 ciudades (18.4\%). En relación con este espacio está, en el extremo sureste, la cuenca del río Mopán con cuatro yacimientos (6.6\%). En esta parte sur de la RBм los sitios arqueológicos, en buena medida, son también las últimas reservas de selva (manchas vegetales en medio de pastizales y terrenos de cultivos). En otros casos, como en Ixlú o Tayasal, la comunidad actual está encima del sitio arqueológico.

La parte oeste de la RBM está representada por siete yacimientos (11.6\%). Solamente uno cuenta con arquitectura expuesta, exhibida por excavaciones arqueológicas recientes (La Joyanca), y el petrograbado de San Diego-La Gloria (número 61 en el mapa de la Figura 2). En los informes de gobernanza del conap, la parte oeste es la más conflictiva, una zona de explotación de petróleo, invasiones y trasiegos ilegales.

La porción suroeste contiene a la cuenca del río Usumacinta, tiene sólo dos referencias (Piedras Negras y Tecolote/Pasadita, 3.4\% de la muestra). Esta parte está un tanto mejor conservada (según ConAP-wsc, 2018), aunque presenta amenazas constantes de invasiones y daños al patrimonio. 


\section{Comparaciones urbanas en los distintos territorios de la RBM}

La composición arquitectónica de los yacimientos en la RBM es distinta. Podemos caracterizar seis condiciones, ubicadas en las zonas este, centro, Los Lagos, oeste, río San Pedro y Usumacinta. En la parte este hay grandes ciudades, de enormes dimensiones, colosales palacios y grupos de edificios que son icono de la arquitectura maya en las tierras bajas centrales; es uno de los territorios con mayor concentración de ciudades prehispánicas en América. Por ejemplo, en esta zona se ubican los 13 centros urbanos más grandes de la RBM: dos súper centros (Tikal y El Mirador), dos centros muy grandes (Naranjo y La Honradez) y nueve centros grandes (Quintana, 2013a). También en ella se encuentran los palacios más espectaculares, como la Acrópolis de Nakum, que tiene 16 patios y 44 edificios, el mayor de la RBM. Hay asimismo diferentes tipologías de edificios para el juego de pelota, incluso los hay dobles y triples (Tikal y La Honradez). Otro elemento distintivo son los edificios conmemorativos, como los "grupos de pirámides gemelas" de Tikal y Yaxhá o los "grupos E” (el más conocido es el grupo E de Uaxactún y el mayor está en Wakná, de 190 m de largo). En la Cuenca Mirador, destacan los grupos de edificios de "tipo tríadico"; hay más de 40 grupos de este tipo grandes y pequeños; los de gran tamaño sobresalen y marcan las ciudades en el horizonte (como el grupo La Danta de El Mirador).

En la parte sur, Cuenca de Los Lagos, hay abundantes sitios que denotan una composición arquitectónica característica del Posclásico: la isla de Topoxté expone edificios del siglo xv, único ejemplo restaurado de esta época cultural en toda la Rвм. Otros sitios contemporáneos son Sacpetén, Ixlú y Tayasal. Tayasal fue la última ciudad maya destruida por los conquistadores (1697) y cuenta con evidencia ocupacional de todas las épocas, ahora con sectores urbanos sobrepuestos por el poblado moderno de San Miguel.

La parte oeste de la RBM tiene otro tipo de composición arquitectónica, no tan gigantesca como las descritas antes. La arquitectura responde al paisaje de otra manera. Aquí las construcciones son dispersas y no llegan a tener grandes dimensiones. Se ubican entre humedales, como en La Corona, o sobre elevaciones aisladas del terreno, como en El Achiotal. Estas ciudades, más bien eran centros de control del territorio (producción de alimentos) y sitios que vigilaban las vías de comercio norte-sur.

Un tanto diferente es la cuenca del río San Pedro Mártir, en la parte suroeste. En este segmento hay varios asentamientos de grandes dimensiones que se ubicaron cerca del río: El Perú, La Joyanca, La Florida, y más al este, El Zotz. Seguramente controlaron esta importante red fluvial que fue una de las vías de acceso al centro del Petén.

Distinta es también la composición arquitectónica en la cuenca del río Usumacinta. En ella las ciudades se amoldaron a las colinas junto al río, formando espectaculares ejemplos de paisaje e integración de patrimonio (a ambos lados de la cuenca, como sucede también en Yaxchilán, en Chiapas). Piedras Negras 
es la ciudad icono de la parte guatemalteca, y una de sus características son los edificios para baños de vapor.

Un elemento común en la mayoría de las ciudades de la muestra es su alta producción de estelas, altares, frisos esculpidos o escalinatas con inscripciones jeroglíficas que cuentan la historia de sus gobernantes. La producción de escritura y monumentos alusivos a los dignatarios es un valor adicional pues, al menos durante 1,169 años, hubo evidencia epigráfica en la RBM (del 300 a.C. en San Bartolo al 869 d.C. en Tikal).

\section{Conclusiones: condiciones generales del patrimonio edificado en la RBM}

Desigualdad de valores cultural y natural en los planes y acciones en la RBM

El Consejo Nacional de Área Protegidas, en su último informe sobre "monitoreo de la gobernabilidad en la RBM", actualización 2017 (ConAP-wCS, 2018), hace referencia a logros y retos a través de 21 indicadores, que sólo se refieren a aspectos naturales y sociales sin tomar en cuenta el patrimonio edificado prehispánico. Lamentablemente el ConAP no considera "indicadores culturales" en su monitoreo del territorio. Por años se ha insistido que la RBM es una reserva especial, que tiene en simbiosis dos patrimonios importantes: el cultural y el natural. El IDAEH, a pesar de ser la institución más antigua (1946) y con mayor presencia física en la RBM, no tiene el liderazgo para hacerse valer en las reuniones del consejo (el IDAEH es uno de los siete miembros del consejo). Es evidente un alejamiento en la consideración entre flora, fauna y patrimonio cultural prehispánico. El MCD y sus dependencias (DGPCN, ${ }^{36}$ IDAEH y otros) deben retomar su rol, hacer alianzas y construir una agenda estratégica para poder recuperar el sector cultura en la RBM.

Ampliación de cobertura —patrimonio mixto— de Tikal

En el año 1979 se declaró a Tikal como patrimonio mixto cultural y natural, el primero en el mundo (UNESCO); 40 años después (2019) es meritorio regresar a la esencia dual de la declaratoria de Tikal para toda la RBм. Una táctica de Guatemala podría ser preparar un plan estratégico para lograr, documentar y justificar, en un mediano plazo, la ampliación de la cobertura de patrimonio mixto de Tikal a toda la RBM. Tikal no hubiese tenido su importancia regional si no hubiera contado con un sistema de ciudades a su alrededor. La RBM es el contenedor actual de la mayor parte del sistema generado por Tikal, por lo tanto, toda la RBM debe ser considerada como categoría de "patrimonio mixto de la Humanidad", condición que la vecina Reserva de la Biósfera de Calakmul adquirió en el año 2014.

36 Dirección General de Patrimonio Cultural y Natural. 


\section{Tikal-Calakmul}

La historia vivida a lo largo de los siglos VI, VII y VIII (aproximadamente del año 504 al 744 d.C.) con Calakmul y Tikal como protagonistas antagónicos es una buena base para que en el siglo xxı (1,300 años después) se pueda escribir o contar una nueva historia común, bajo el marco de una Gran Reserva de Biósfera de patrimonio mixto.

Falta un plan estratégico del sector cultura en la RBM

El primer plan de Petén en Tikal (1972) tuvo un enfoque mixto en donde predominó el aspecto cultural. En los nuevos planes maestros (a partir de 1990) predomina el aspecto natural. Ahora se utiliza una metodología diseñada para áreas protegidas naturales (PCA y EAPC), liderada por ONGS, locales o internacionales (TNC, WCS, CATIE, UICN y otras). Es claro el predominio del aspecto natural. Lo concerniente a la cultura es insertado dentro del esquema metodológico, sin mayor protagonismo y coherencia.

Históricamente, en la RBM, el MCD-IDAEH ha relegado en otras instituciones la ejecución de proyectos de inversión en el patrimonio cultural: entre 1979-1985 el Proyecto Nacional Tikal fue administrado por el Ministerio de Comunicaciones, Transportes y Obras Públicas (fondos BCIE); ${ }^{37}$ entre 1998-2007 el Programa de Desarrollo Sostenible (PDS) de Petén intervino en Yaxhá y fue administrado por la SEGEPLAN ${ }^{38}$ y luego por el Ministerio de Agricultura (fondos BID). ${ }^{39}$

Fortalezas de patrimonio mixto en la RBM

Una estrategia podría ser fortalecer un grupo de tres zonas núcleo adyacentes una a la otra en la parte central de la RBM: Biotopo San Miguel-La Palotada-El Zotz, al oeste, Tikal, al centro, y Yaxhá, Nakum, Naranjo, al este (129.6 ha y más de $65 \mathrm{~km}$ de largo oeste-este). Este espacio del "Gran Tikal" podría ser el primer detonador de una ampliación de categoría de manejo del patrimonio mixto. Por el momento, la parte faltante por "activar" es la porción oeste, administrada por la USAC (Biotopo El Zotz).

Otra fortaleza sería activar la franja Norte, vecina a la Reserva de la Biósfera de Calakmul, el Parque Nacional Mirador-Río Azul-Biotopo Dos Lagunas, un rectángulo de $100 \mathrm{~km}$ de largo con categoría de zona núcleo de gran potencial, sin un esquema de funcionamiento multidisciplinario fortalecido. Igualmente, en esta acción debería la USAC tener un mayor protagonismo.

\footnotetext{
37 Banco Centroamericano de Integración Económica.

38 Secretaría de Planificación y Programación de la Presidencia, Guatemala.

39 Banco Interamericano de Desarrollo.
} 
Los corredores biológicos (dos), están considerados como zonas de usos múltiples, sin definición y sin acciones concretas para su función de corredor. Debe haber conexión entre parques. También hace falta un Plan Maestro para la zum. Este sector de la RBM es el único que no cuenta con un plan definido como lo tienen ahora: los parques, los biotopos y las zonas de amortiguamiento.

Una estrategia debería ser la conservación preventiva del patrimonio mixto de la RBM

Las lecciones aprendidas de las dos reservas de la biósfera, Calakmul y Maya, deben ser aprovechadas, con 30 años de experiencias en Calakmul y 60 en Tikal. Es necesario un plan estratégico que defina las prioridades de atención a los patrimonios con equidad, además de un plan de conservación preventivo (programa de rescate) para el patrimonio edificado que pueda atender edificios en peligro y ayudar a revertir condiciones de inestabilidad causadas por el saqueo (documentación y relleno de saqueos).

Condición general del patrimonio edificado:

territorio en estado de emergencia

La muestra analizada de 60 ciudades es sólo un reflejo de la calidad y complejidad arquitectónica de la RBM. La verdadera situación de cientos de yacimientos es el abandono. La condición del patrimonio edificado maya en su interior continúa en peligro de perderse. Hace más de 30 años, en la publicación de 1989, se escribió: "Debido a la magnitud de los daños encontrados, que sobrepasan los recursos económicos y técnicos de Guatemala, es necesario tener una panorámica general de la situación real de los sitios arqueológicos y localizar entre ellos los de una mayor necesidad de intervención para tratar de garantizar su preservación" (Siller y Quintana, 1989: 53). En la muestra, unos sitios sólo cuentan con vigilancia y otros están sin atención alguna. En otro grupo, la "estabilidad" depende de un proyecto de investigación arqueológica (fondos de universidades extranjeras), que no atiende precisamente las prioridades de rescate del patrimonio edificado.

Los datos del Programa de Rescate (2005), señalan en un resumen los "indicadores de edificios en peligro", siendo un esbozo de las prioridades de atención de este elemento cultural en la RBM (Quintana, 2013a: 269, Cuadro 7). El resumen identificó 11 sitios prioritarios (La Muralla, Naachtún, El Zotz, Río Azul, Kinal, Chochkitam, La Honradez, Xultún, Holmul, Tzikintzakán y Chilonché); 14 años después, nueve sitios siguen sin atención, con arquitectura en peligro de colapso (La Muralla, Naachtún, El Zotz, Río Azul, Kinal, Chochkitam, Xultún, Holmul y Tzikintzakán). En dos hubo una corta actividad: La Honradez y Chilonché, pero posteriormente ya no fueron atendidos. 
1) Sitios con arquitectura expuesta, en pie o visible

Siguiendo los dos indicadores publicados en 1989 podemos determinar ciertos avances en relación con la intervención de arquitectura expuesta. De la muestra analizada (60 ciudades) podemos saber que 45 sitios cuentan con arquitectura visible o expuesta, y 15 sitios no la presentan. De 60 ciudades se han realizado acciones restaurativas en 19 yacimientos. La totalidad de edificios con elementos arquitectónicos expuestos suman 305 inmuebles, de los cuales se han realizado intervenciones en 233. En algunos de ellos fueron acciones necesarias, en otros no. En unos se trabajó en forma integral, en otros sólo las fachadas. En algunos se respetaron los principios internacionales de conservación, en otros se hicieron reconstrucciones.

También sabemos que de 60 ciudades que conforman la muestra, 20 tienen intervenciones de conservación en su interior, túneles de mantenimiento (frisos de El Diablo, friso y mascarón en Holmul, mascarón en Cival, las pinturas murales de San Bartolo, las tumbas de Río Azul, varios túneles en Tikal y otros sitios). Hay ciudades como Holmul, que cuenta con arquitectura intervenida, pero la ciudad presenta varios edificios con arquitectura expuesta sin atención (siete edificios en peligro), por esa razón la prioridad no ha sido atendida. Sin embargo, la condición general de la mayoría del patrimonio edificado de la RBM — sitios y edificios- mantiene tres condicionantes: 1 ) abandono, 2) vegetación sin control sobre los edificios y 3 ) saqueo. La vegetación sin control sobre los edificios en la selva siempre verde es un factor importante de daños al patrimonio edificado; generaciones de árboles han nacido, crecido y muerto sobre muros y techos de edificios en ruinas.

\section{2) Cantidad de saqueos}

Todos los sitios de la muestra presentan depredaciones. En la gran mayoría de ellos, sus edificios siguen siendo abiertos por esta actividad ilícita. De 60 sitios de la muestra, 52 permanecen sin relleno de saqueos. Los datos de saqueo sobre los bienes inmuebles también dan información sobre la presencia humana en la zona; pueden considerarse como "rastreadores" de la condición del patrimonio cultural. Por ejemplo, si en el monitoreo de un edificio diagnóstico, éste indica que hay más saqueos que los reportados y publicados, quiere decir que hay un mal manejo del parque o la zona. El saqueo es una actividad ilegal pero existente. Además del saqueo arqueológico, hay cacería furtiva, se corta madera, se provocan incendios y se realizan otras actividades ilegales en la RBM.

\section{Cooperación y trabajos multi-institucionales}

Si existiera un plan estratégico de la RBM, las autoridades responsables del patrimonio podrían solicitar de nuevo el apoyo de la cooperación internacional. 
Es claro que el Estado solo no puede atenderle. Un ejemplo exitoso fue la cooperación alemana (1994-2006) en el Triángulo Cultural Yaxhá, Nakum, Naranjo, área que cuenta desde el año 2002 con su propio esquema de funcionamiento para garantizar el seguimiento y su mantenimiento. En el Programa Selva Maya, el gobierno alemán continúa, desde el año 2011, apoyando la conservación de la selva, ahora en forma más extensa abarcando partes de la RBM, Belice, Quintana Roo y Campeche.

La universidad estatal (UASC) administra dentro de la RBM varios biotopos, zonas núcleo que pueden activarse como laboratorios de aplicación de nuevas tecnologías y trabajos multidisciplinarios. Un papel fundamental de esta casa de estudios superiores es la investigación científica, el entrenamiento y capacitación de sus alumnos. La UASC debería impulsar una especie de "Escuelas Taller" en las ciencias del rescate, conservación y manejo de patrimonio mixto.

La iniciativa privada puede colaborar también en las tareas de inversión, rescate e investigación de los recursos de la RBM, como lo ha realizado desde el año 2006 la Fundación Patrimonio Cultural y Natural Maya. El apoyo financiero de la iniciativa privada, dirigida a recuperar bienes inmuebles identificados como prioritarios, debe de impulsarse. Varios proyectos de investigación arqueológica han sido apoyados, por la iniciativa Lidar-Pacunam, al contar con tecnología de cartografía aérea de alta calidad. En la muestra de 60 sitios, 22 ciudades cuentan con esta característica de documentación cartográfica.

Fragilidad de los proyectos de investigación de universidades extranjeras en la RBM

Aquí prevalece la investigación arqueológica académica, el comprobar teorías de profesores y el entrenamiento de alumnos (para tesis de maestría y doctorado) sin considerar, en la mayoría de los proyectos de investigación, aspectos de ayuda a la conservación del patrimonio. Un patrimonio que muchas veces se encuentra en peligro de colapso y que requiere medidas de rescate y restauración, de bienes inmuebles que ahora ya están identificados y priorizados (Quintana y Wurster, 2001; Quintana, 2013a).

El Estado no posee una estrategia para recuperar el patrimonio edificado en peligro de colapso. Debe prevalecer en las acciones de investigación arqueológica un código ético en favor de la conservación del patrimonio edificado expuesto y en peligro. El corpus de acciones restaurativas en la RBM (233 edificios) es importante y requiere atención. Para ello el Estado debe elaborar un plan estratégico de mantenimiento, conservación, uso y presentación de este patrimonio edificado.

Participación de las comunidades vecinas

La inversión en el sector de cultura se justifica al poder asociarlo con el desarrollo de actividades de turismo y con los programas de conservación de la 
naturaleza. Es importante integrar, capacitar y educar a los poblados vecinos en los proyectos arqueológicos y en los proyectos de conservación de la naturaleza, no como observadores temporales sino como partes de una fuente de trabajo digno y perdurable. Estas acciones son más prioritarias en la parte sur de la RBM, territorios ZAM y áreas protegidas de concesiones forestales comunitarias (ZUM).

\section{Bibliografía}

CONAP

2016 Reserva de la Biósfera Maya. Plan Maestro. Segunda actualización a diciembre 2015 (Documentos Técnicos núms. 20, 21, 22 y 23-2016).

Consejo Nacional de Áreas Protegidas (ConAP) y Wildlife Conservation Society (wCS)

2018 Monitoreo de la gobernabilidad en la Reserva de la Biósfera Maya. Actualización a 2017, con apoyo de la Agencia de los Estados Unidos para el Desarrollo Internacional (USDAI) y el U.S. Department of the Interior's International Technical Assistance Program (USDOI/ITAP).

Morley, Sylvanus G.

1938 The Inscriptions of Peten. Washington, D.C.: Carnegie Institution of Washington (Publication 437), 5 Vols.

Quintana, Oscar

2013a Ciudades mayas del noreste de Petén, Guatemala: composición arquitectónica y conservación. Prólogo de Carlos Batzin Chojoj, contribuciones de Raúl Noriega, Breitner González y Telma Tobar. Wiesbaden: Reichert Verlag (Forshungen zur Archäologie Aussereuropäischer Kulturen, Band 11).

2013b "Resultados del proyecto guatemalteco-alemán en el noreste de Petén, Guatemala, 1994-2006", Anuario de la Dirección General del Patrimonio Cultural y Natural. Antropología e Historia, III (12): 35-66.

2014 "Tikal y sus vecinos, acontecimientos históricos en el noreste de Petén, 1834-1987”, Anuario de la Dirección General del Patrimonio Cultural y Natural. Antropología e Historia, III (13): 7-75.

2015 “Ciudades Mayas, recorridos por el noreste de Petén”, Anuario de la Dirección General del Patrimonio Cultural y Natural. Antropología e Historia, III (14): 77-152.

Quintana, Oscar y Juan Antonio Siller Camacho

1992 "Patrimonio cultural, natural y ecodesarrollo: Yaxhá, Nakum y Naranjo, Petén, Guatemala”, Cuadernos de Arquitectura Mesoamericana, 16: 55-69.

2019 "Recorridos y evaluación de condición del patrimonio edificado en el cuadrante noreste de Petén, Guatemala, 1987-2018”, Anuario de la Dirección del Patrimonio Cultural y Natural. Antropología e Historia de Guatemala, III (18): 131-200.

Quintana, Oscar y Wolfgang W. Wurster

2001 Ciudades Mayas en el noreste de Peten, Guatemala, un estudio urbanístico compa- 
rativo. Con planos y dibujos de Raúl Noriega. Mainz am Rhein: Verlag Philipp von Zabern (Materialien zur Allgemeinen und Vergleichenden Archäologie Band, 59).

Siller, Juan Antonio

1991a "Experiencias obtenidas a través de los viajes de reconocimiento arquitectónico del Seminario de Arquitectura Prehispánica”, Cuadernos de Arquitectura Mesoamericana, 12: 83-90.

1991b "Viaje de reconocimiento arquitectónico en el Petén, Guatemala, febreromarzo 1989. Consideraciones generales", Cuadernos de Arquitectura Mesoamericana, 15: 67-74.

Siller, Juan Antonio y Oscar Quintana

1989 "Reconocimiento arquitectónico de sitios arqueológicos en Petén", Cuadernos de Arquitectura Mesoamericana, 11: 51-83.

UNESCO

2012 Gran Atlas del Patrimonio Mundial. Barcelona: Editorial Planeta.

Oscar Antonio Quintana Samayoa Guatemalteco. Arquitecto por la Universidad de San Carlos de Guatemala, maestro en restauración de monumentos en la Universidad Técnica de Múnich y doctor en composición arquitectónica por la Universidad Politécnica de Valencia. Adscrito a la Facultad de Arquitectura de la Universidad de San Carlos de Guatemala, su especialidad es la composición arquitectónica prehispánica y la conservación de edificios. Actualmente es consultor del programa trinacional "Selva Maya". Entre sus publicaciones más recientes se encuentran "Recorridos y evaluación de condición del patrimonio edificado en el cuadrante noreste de Petén, Guatemala, 1987-2018”, “El noreste de El Petén, aportes al urbanismo prehispánico" y "Ciudades mayas, recorridos por el noreste de Petén. Resultados 2001-2013”, la primera en coautoría.

oscar_quintanasamayoa@yahoo.com

Juan Antonio Siller Camacho. Mexicano. Arquitecto por la Facultad de Arquitectura de la Universidad Nacional Autónoma de México y arqueólogo por la Escuela Nacional de Antropología e Historia, maestro en Restauración de Monumentos Históricos y Museología por la Escuela Nacional de Conservación, Restauración y Museografía, doctor en Arquitectura Prehispánica por la Facultad de Arquitectura de la unAm. Adscrito al Posgrado de la Facultad de Arquitectura de esta Universidad, se ha especializado en la arquitectura y el urbanismo prehispánico, la conservación y restauración de monumentos arqueológicos e históricos, entre otros aspectos. Entre sus proyectos en curso están "Las misiones jesuitas de Baja 
California", "Los itinerarios culturales del Galeón de Manila" y "Los paisajes culturales de la Cuenca de México". Entre sus últimas publicaciones se encuentran "Recorridos y evaluación de condición del patrimonio edificado en el cuadrante noreste de Petén, Guatemala, 1987-2018”, El itinerario cultural del Galeón de Manila, préstamos culturales y "El Jardín Borda en el paisaje histórico de Cuernavaca", en coautoría.

juanantoniosillercamacho@gmail.com 